\title{
Zoledronic acid promotes osteoclasts ferroptosis by inhibiting FBX09-mediated p53 ubiquitination and degradation
}

\author{
Xingzhou Qu ${ }^{1}$, Zhaoqi Sun ${ }^{1}$, Yang Wang ${ }^{\text {Corresp., } 1}{ }^{\text {, shan Ong Hui }}{ }^{\text {Corresp. } 1}$ \\ ${ }^{1}$ Department of Oral and Maxillofacial-Head \& Neck Oncology,, Ninth People's Hospital Affiliated to Shanghai Jiao Tong University School of Medicine, SH, \\ Shanghai, China \\ Corresponding Authors: Yang Wang, shan Ong Hui \\ Email address: 115078@sh9hospital.org.cn, 117069@sh9hospital.org.cn
}

Bisphosphonates (BPs)-related osteonecrosis of jaw (BRONJ) is a severe complication of the long-term administration of BPs. The development of BRONJ is associated with the cell death of osteoclasts, but the underlying mechanism remains unclear. In the current study, the role of Zoledronic acid (ZA), a kind of bisphosphonates, in suppressing the growth of osteoclasts was investigated and its underlying mechanism was explored. The role of ZA in regulating osteoclasts function was evaluated in the RANKL-induced cell model. Cell viability was assessed by cell counting kit-8 (CCK-8) assay and fluorescein diacetate (FDA)staining. We confirmed that ZA treatment suppressed cell viability of osteoclasts. Furthermore, ZA treatment led to osteoclasts death by facilitating osteoclasts ferroptosis, as evidenced by increased $\mathrm{Fe}^{2+}$, ROS, and malonyldialdehyde (MDA) level, and decreased glutathione peroxidase 4 (GPX4) and glutathione (GSH) level. Next, the gene expression profiles of alendronate- and risedronate-treated osteoclasts were obtained from Gene Expression Omnibus (GEO) dataset, and 18 differentially expressed genes were identified using venn diagram analysis. Among these 18 genes, the expression of F-box protein 9 (FBXO9) was inhibited by ZA treatment. Knockdown of FBXO9 resulted in osteoclasts ferroptosis. More important, FBXO9 overexpression repressed the effect of ZA on regulating osteoclasts ferroptosis. Mechanistically, FBXO9 interacted with p53 and decreased the protein stability of p53. Collectively, our study showed that ZA induced osteoclast cells ferroptosis by triggering FBX09-mediated p53 ubiquitination and degradation. 
$1 \quad$ Zoledronic acid promotes osteoclasts ferroptosis by inhibiting FBXO9mediated p53 ubiquitination and degradation

3 Xingzhou Qu, Zhaoqi Sun, Yang Wang*, Hui shan Ong*

4 Department of Oral and Maxillofacial-Head \& Neck Oncology, Ninth People's Hospital Affiliated

5 to Shanghai Jiao Tong University School of Medicine, Shanghai 200011, China

*Correspondence author: Hui shan Ong, Department of Oral and Maxillofacial-Head \& Neck

7 Oncology, Ninth People's Hospital Affiliated to Shanghai Jiao Tong University School of

8 Medicine, No. 639, Zhizaoju Road, Shanghai 200011, China. Tel: 86-21-23271699-5160. Email:

9 117069@sh9hospital.org.cn Yang Wang, Department of Oral and Maxillofacial-Head \& Neck

10 Oncology, Ninth People's Hospital Affiliated to Shanghai Jiao Tong University School of

Medicine, No. 639, Zhizaoju Road, Shanghai 200011, China. Tel: 86-21-23271699-5160. Email:

Email: 115078@sh9hospital.org.cn 


\section{Abstract}

Bisphosphonates (BPs)-related osteonecrosis of jaw (BRONJ) is a severe complication of the longterm administration of BPs. The development of BRONJ is associated with the cell death of osteoclasts, but the underlying mechanism remains unclear. In the current study, the role of Zoledronic acid (ZA), a kind of bisphosphonates, in suppressing the growth of osteoclasts was investigated and its underlying mechanism was explored. The role of ZA in regulating osteoclasts function was evaluated in the RANKL-induced cell model. Cell viability was assessed by cell counting kit-8 (CCK-8) assay and fluorescein diacetate (FDA)-staining. We confirmed that ZA treatment suppressed cell viability of osteoclasts. Furthermore, ZA treatment led to osteoclasts death by facilitating osteoclasts ferroptosis, as evidenced by increased $\mathrm{Fe}^{2+}$, ROS, and malonyldialdehyde (MDA) level, and decreased glutathione peroxidase 4 (GPX4) and glutathione (GSH) level. Next, the gene expression profiles of alendronate- and risedronate-treated osteoclasts were obtained from Gene Expression Omnibus (GEO) dataset, and 18 differentially expressed genes were identified using venn diagram analysis. Among these 18 genes, the expression of Fbox protein 9 (FBXO9) was inhibited by ZA treatment. Knockdown of FBXO9 resulted in osteoclasts ferroptosis. More important, FBXO9 overexpression repressed the effect of ZA on regulating osteoclasts ferroptosis. Mechanistically, FBXO9 interacted with p53 and decreased the protein stability of $\mathrm{p} 53$. Collectively, our study showed that ZA induced osteoclast cells ferroptosis by triggering FBXO9-mediated p53 ubiquitination and degradation.

Keywords: Bisphosphonates related osteonecrosis of jaw; Zoledronic acid; ferroptosis; FBXO9; 
p53

44

45

46

47

Bisphosphonates (BPs) inhibit osteoclast activity and disrupt osteoclast mediated bone resorption $[1,2]$. BPs are widely used in the treatment of bone metastasis cancer[3], osteoporosis[4], and multiple myeloma[5]. BRONJ is an injury of the jaw that affects patients treated with BPs. Since it was reported in 2003, BRONJ has been considered as a common and important adverse side effect of BPs treatment, especially nitrogen-containing bisphosphonates (Residronate, Alendronate, and Zoledronic acid(ZA))[6]. There are various hypotheses for the development of BRONJ, the most recognized hypothesis was bone remodeling suppression. Although significant progress has been made in prevention and treatment of BRONJ base on the hypotheses, the mechanisms underlying the development of BRONJ remain unclear.

Osteoclasts, members of the monocyte/macrophage hematopoietic, play an important role in the progression of bone remodeling[8]. RAW264.7 cells and bone marrow-derived macrophages (BMDMs) can be induced into osteoclasts by receptor activator of nuclear factor- $\mathrm{kB}$ ligand (RANKL), and has been widely used as a cell model for the study of osteoclast related diseases in vitro[9]. The number and the resorptive function of osteoclasts were usually increased during the process of bone remodeling [10]. Therefore, osteoclast is one of the core targets for the treatment of osteoporosis and other bone-remodeling-related diseases. It is well known that ZA can lead to a stronger inhibition of osteoclasts differentiation and induces the apoptosis of osteoclasts[11], while the underlying mechanism of ZA in the function of osteoclasts reminds unclear.

Ferroptosis is a recently identified type of iron-mediated cell death. Unlike other forms of 
64 programmed cell death, such as apoptosis and necroptosis, ferroptosis does not involve the 65 activation of caspase protein[12, 13]. It is characterized by an increased level of lipid peroxidation

66

67

products and reactive oxygen species (ROS). The dysregulation of ferroptosis has been related to many pathological processes, such as cancer[14], neurodegenerative diseases[15], and inflammation-related diseases[16]. More and more studies showed that ferroptosis contribute to the development of BRONJ. Jose et al. found that the levels of MDA, GSSG, and 8-oxo-dG and the GSSG/GSH ratio in serum and saliva were significantly higher in patients with BRONJ compared with controls[17]. Ma et al. demonstrated that melatonin suppresses osteoblast ferroptosis and improved the osteogenic capacity of MC3T3-E1 by activating the Nrf2/HO-1 pathway[18]. However, whether ferroptosis was involved in the osteoclasts differentiation and death induced by ZA is still unknown. In the current study, we showed that ZA inhibits the osteoclasts viability in a dose-dependent manner. For the first time, we showed that ZA promotes the ferroptosis of osteoclast by increasing the protein stability of p53. ZA-induced downregulation of ubiquitin E3 ligase FBXO9, and FBXO9 overexpression restores cell viability inhibition of osteoclast induced by ZA. Moreover, FBXO9 facilitates ubiquitination-mediated degradation of p53.

\section{Materials and methods}

\section{Cell culture}

RAW264.7 cells were purchased from the ATCC (TIB-71, Manassas, VA, USA) and cultured in alpha-Modified Eagle's Medium ( $\alpha$-MEM, Gibco, USA) with $100 \mathrm{U} / \mathrm{ml}$ penicillin, and $100 \mu \mathrm{g} / \mathrm{ml}$ 
85

86

87

streptomycin at $37{ }^{\circ} \mathrm{C}$ in a humidified atmosphere containing $5 \% \mathrm{CO}_{2}$ and $95 \%$ air. To BMDMs, bone marrow cells were purchased from the ATCC (CRL-2420, Manassas, VA, USA) and cultured in RPMI 1640 medium (Thermo Fisher Scientific, Waltham, MA, USA) for 6 days with $100 \mathrm{U} / \mathrm{ml}$ penicillin, $100 \mu \mathrm{g} / \mathrm{ml}$ streptomycin, $10 \% \mathrm{FBS}$, and $10 \mathrm{ng} / \mathrm{mL}$ recombinant mouse macrophage colony-stimulating factor (PeproTech). For osteoclast formation assay.RAW264.7 cells were seeded in 12 -well plate $\mathrm{s}\left(1 \times 10^{4}\right.$ cells/well) supplemented with $50 \mathrm{ng} / \mathrm{ml}$ RANKL (R\&D Systems.) for 6 days. BMDMs $\left(1 \times 10^{4}\right.$ cells/well $)$ were cultured in the presence of M-CSF $(10 \mathrm{ng} / \mathrm{mL})$ and RANKL (50 ng/mL) for 6 days.

\section{TRAP staining}

TRAP histochemical staining was performed to confirm the osteoclast as previously described[25], by using an acid phosphatase, leukocyte (TRAP) kit (Sigma-Aldrich). Brifely, $1 \times 10^{5}$ BMDMs or RAW264.7 induced osteoclasts were then fixed in 10\% neutral-buffered formalin (NBF) solution for 20 minutes, then the NBF solution was replaced with TRAP staining solution and $0.1 \%$ Fast Red AS TR salt at RT. After 45 minutes cells were washed with $1 \times$ PBS for three times and imaged.

\section{Measurement of cell viability}

Cell viability of BMDMs and RAW264.7 was assessed by using a CCK-8 and FDA assay (Dojindo, Japan). For CCK-8 assay, BMDMs and RAW264.7 cells $\left(1 \times 10^{4}\right.$ cells per well) were seeded in 96-well plates for 24 hours, then cells were treated with different doses of ZA $(5,10$, and $50 \mu \mathrm{M})$ for $48 \mathrm{~h}$. To analyze the cells death of osteoclast, BMDMs, and RAW264.7 $\left(5 \times 10^{3}\right.$ cells per well) were treated with $10 \mu \mathrm{M}$ of ZVAD-FMK, $2 \mu \mathrm{M}$ of Fer-1, or $10 \mu \mathrm{M}$ of necrostatin-1, 
106 for $48 \mathrm{~h}$ with or without ZA $(50 \mu \mathrm{M})$. Then, a total $10 \mu \mathrm{L}$ of CCK-8 reagent was added to each well

107 additional $4 \mathrm{~h}$ at $37^{\circ} \mathrm{C}$ with $5 \% \mathrm{CO}_{2}$, and the absorbance at $450 \mathrm{~nm}$ of each well was assayed using 108 a microplate reader (BioTek Instruments). For FDA assay, after culture with different dose ZA (5, 10910 , and $50 \mu \mathrm{M})$ for 48h, BMDMs and RAW264.7 cells were treated with $10 \mu$ of FDA solution (5 $110 \mathrm{mg} / \mathrm{mL}$; Invitrogen, CA, USA) at $37^{\circ} \mathrm{C}$ with $5 \% \mathrm{CO}_{2}$ for 20 minutes, then cells images were 111 obtained using a fluorescence microscope (Olympus Corporation, Japan).

\section{$112 \mathbf{F e}^{2+}$ concentration}

113 The concentration of Ferrous iron $\left(\mathrm{Fe}^{2+}\right)$ in BMDMs and RAW264.7 cells in the presence or 114 absence of ZA $(50 \mu \mathrm{M})$ was assessed using an iron assay kit (MAK025, Sigma-Aldrich, MO, USA) 115 as the manufacturer's instructions. Briefly, cell samples were incubated with $10 \mu \mathrm{L}$ of iron reducer 116 for 30 minutes at RT, then $100 \mu \mathrm{L}$ iron probe was added to trigger the reaction; thus, the absorbance 117 was measured at $593 \mathrm{~nm}$.

\section{Lipid reactive oxygen species (ROS) assay}

Lipid ROS level in BMDMs and RAW264.7 cells in the presence or absence of ZA $(50 \mu \mathrm{M})$ was

assayed using C11-BODIPY (Invitrogen), a fluorescent-labelled oxidation sensitive probe. In brief, BMDMs and RAW264.7 cells were seeded in 24 -well plates $\left(5 \times 10^{5} /\right.$ well $)$ and treated with ZA $(50 \mu \mathrm{M})$ with or without FBXO9 overexpression for 48h, then BMDMs and RAW264.7 cells were cultured with $\mathrm{C} 11$-BODIPY probe with a final concentration of $1 \mu \mathrm{M}$ in at $37^{\circ} \mathrm{C}$ with $5 \% \mathrm{CO}_{2}$ for 30 minutes, then the Lipid ROS levels were assayed using flow cytometer.

\section{MDA and GSH content}

MDA in BMDMs and RAW264.7 cells was analyzed using a lipid peroxidation assay kit 
127 (ab118970, Abcam) in the presence or absence of ZA $(50 \mu \mathrm{M})$. GSH content in BMDMs and

128 RAW264.7 cells was assayed using a Glutathione Assay Kit (ab65322, Abcam) according to the

129 standard protocol. Briefly, cell supernatant, 5,5' -dithio-bis 2-nitrobenzoic acid solution and the 130 reagents of kits were mixed together and incubated at RT for 10 minutes, then NADPH was added 131 into this system to trigger the reaction. the absorbance of 5-thio-2-nitrobenzoic acid was detected 132 at $412 \mathrm{~nm}$.

\section{Transient transfection of FBXO9 or si-FBXO9}

134 The recombinant plasmids pcDNA-FBXO9 containing FBXO9 cDNA were sub-cloned into 135 pcDNA3.1 vector via EcoR V/Hind III sites. To overexpress FBXO9, the pcDNA-FBXO9 was 136 transfected into BMDMs cells using Lipofectamine 2000. FBXO9 Knockdown and 137 transfection were performed according to the manufacturer's instructions. Briefly, cells were transfected with $10 \mathrm{nM}$ of si-FBXO9 RNA (sense AUCAGAAUGACAAUCUUCCUCU, antisense GGAAGAUUGUCAUUCUGAUGCU

140 ) or si-Control RNA (sense CAGUCGCGUUUGCGACUGGUU, antisense CCAGUCG141 CAAACGCGACUGUU), and the cell was induced by M-CSF (10 ng/mL) and RANKL (50 $142 \mathrm{ng} / \mathrm{mL}$ ) for further experiment.

\section{Quantitative real-time PCR (qPCR)}

After treatment with $50 \mu \mathrm{M}$ of ZA for $48 \mathrm{~h}$, total RNA was isolated with Trizol reagent (Sigma-

Aldrich) as instructed by the manufacturer. Reverse transcriptional PCR was carried out using the

SMART PCR cDNA Synthesis Kits (Clontech). qPCR was carried out on ABI 7500 RealTime

PCR System (Applied Biosystem) with powerup SYBR green Mix (ThermoFisher). The fold 
148 changes of RNA transcripts were calculated by the $2^{-\Delta \Delta \mathrm{Ct}}$ method and the $18 \mathrm{~s}$ was used as a

149 reference gene. The qPCR primer pairs in Table 1

150 Western blotting analysis

151 BMDMs-induced osteoclast $\left(1 \times 10^{5}\right.$ cells/well in 12 -well plates $)$ were treated with or without $152 \mathrm{ZA}(50 \mu \mathrm{M})$ for $48 \mathrm{~h}$, the total protein was isolated using RIPA Buffer (Solarbio, Beijing, China), 153 and total protein concentration was quantified by BCA protein assay kit (Beyotime). $20 \mu \mathrm{g}$ of protein were separated by $8 \%$ SDS-PAGE and transferred to PVDF membranes (Merck Millipore, Billerica, MA, USA). After blocking with $5 \%$ bovine serum albumin or $5 \%$ nonfat milk, the membranes were incubated with anti-FBXO9 (1:1000, PA5-25475, Thermo Fisher Scientific), p53(1.0 $\mu \mathrm{g} / \mathrm{mL}$, MA5-14067, Thermo Fisher Scientific), ubiquitin (1:2000, ab134953, Abcam), and GAPDH (1:5000, MA1-16757, Thermo Fisher Scientific) overnight at $4{ }^{\circ} \mathrm{C}$. Then the membranes were treated with HRP-conjugated anti-mouse or rabbit secondary antibody (1:5000) for $1 \mathrm{~h}$ at room temperature.

\section{Co-immunoprecipitation (Co-IP)}

BMDMs-induced osteoclast were lysed using NP40 buffer $(10 \mathrm{mM}$ Tris- $\mathrm{HCl}$ at $\mathrm{pH} 8.0,140 \mathrm{mM}$

$\mathrm{NaCl}, 1.5 \mathrm{mM} \mathrm{MgCl} 2,0.5 \%$ Nonidet-P40, $20 \mathrm{mM}$ dithiothreitol, $500 \mathrm{U} / \mathrm{mL}$ RNAsin, and 0.5\%

[w/v] deoxycholate), cell lysates were incubated with FBXO9 $(2 \mu \mathrm{g} / \mathrm{ml}$; PA5-25475) or p53 (2

$\mu \mathrm{g} / \mathrm{ml}$; MA5-14067) antibody for 4h, then Protein A/G beads (Thermo Fisher Scientific) were added to the IP reactions and left rotating overnight at $4^{\circ} \mathrm{C}$, then beads were washed by PBS containing protein inhibitors for three times, then the immunoprecipitates were analyzed using western blotting with FBXO9 antibody or p53 antibody. 
169

170

171

172

173

174

175

176

177

178

179

180

181

182

183

184

185

186

187

188

189

\section{Statistical analysis}

All the data are shown as the mean \pm standard error of mean (SEM) from three independent experiments. Statistical analysis was carried out using SPS 19.0 software (IBM Corp., NY. USA). The significance between two groups was analyzed using one-way ANOVA followed by Tukey-Kramer multiple comparisons test or unpaired Student's $t$-test. $p<0.05$ was considered to indicate a statistically significant difference.

\section{Results}

\section{ZA treatment facilitated the ferroptosis of osteoclasts}

To investigate the function of ZA on osteoclasts, RAW264.7 cells and bone marrow-derived macrophages (BMDMs) were pre-treated with RANKL for 6 days followed by ZA treatment in different concentrations $(5,10$, and $50 \mathrm{uM})$ for 48 hours, the cell model was confirmed byTRAP staining (Figure1 A and B). Cell viability was analyzed by CCK-8 assay. As shown in Figure1 C and D, ZA treatment suppressed cell viability in a dose-dependent manner. The results from FDA staining also showed that the effect of ZA on promoting cell viability of osteoclasts (Figure 1E and F). Impressively, the CCK-8 assay results showed that cell death of osteoclasts induced by ZA treatment was obviously blocked by ferrostatin-1 (Fer-1, a specific inhibitor of ferroptosis) but not necrostatin-1 (a specific inhibitor of necroptosis) and ZVAD-FMK (a specific inhibitor of apoptosis) (Figure $1 \mathrm{G}$ and $\mathrm{H}$ ).

To define the role of ZA in the ferroptosis of osteoclasts, the ferroptosis signaling was evaluated in osteoclasts after ZA treatment. As shown in Figure 2A-C and F-H, ZA treatment markedly 
190

191

192

193

194

195

196

197

198

199

200

201

202

203

204

205

206

207

208

209

210

increased the $\mathrm{Fe}^{2+}$ level, MDA content, and ROS level in a dose-dependent manner in osteoclasts, differentiated from RAW264.7 cells and BMDMs, suggesting the promotion of ferroptosis signaling in osteoclasts treated by ZA. Besides, ZA treatment also suppressed the levels of Gpx4 and GSH in a dose-dependent manner in osteoclasts (Figure 2D, E, I, and J). These results demonstrate that ZA treatment facilitates the ferroptosis of osteoclasts.

\section{FBXO9 was downregulated in osteoclasts after ZA treatment}

To investigate the mechanism underlying ZA-induced osteoclasts ferroptosis, the differentially expressed genes (DEGs) of osteoclasts induced by bisphosphonates alendronate- and risedronatetreatment were obtained from GSE63009, and the common DEGs were identified by venn diagram analysis. As shown in Figure 3A, eighteen common genes were identified (CFAP53, COL14A1, ARSJ, ABCA9, CXorf57, GPR22, STXBP5L, MSANTD4, RRP15, UGT1A2, IRF4, TFAP2D, TRHDE, ASMT, CAPS, COMMD10, VSTM4, FBXO9). The level of these 18 genes was evaluated in osteoclasts treated with or without ZA using qPCR analysis. Figure 3B and C showed that only FBXO9 was significantly decreased in osteoclasts after treatment with ZA. Similar to the qPCR results, the results from western blotting showed that the expression of FBXO9 was obviously decreased in osteoclasts after treatment with ZA. These results indicate that the FBXO9 was downregulated by ZA treatment (Figure 3D).

\section{FBXO9 inhibition facilitated the ferroptosis of osteoclasts}

To investigate the function of FBXO9 on osteoclasts, the expression of FBXO9 was downregulated by si-FBXO9 in BMDMs-differentiated osteoclasts. As shown in Figure 4 A-B, the expression of FBXO9 was significantly decreased by si-FBXO9. Cell viability of osteoclasts was 
211 significantly decreased in the FBXO9 knockdown group compared with the control group (Figure

212 4C). The results from FDA staining also showed the effect of FBXO9 on inhibiting cell viability

213 (Figure 4D and E).

214 To investigate the role of FBXO9 in ferroptosis of osteoclasts, the ferroptosis signaling was

215 evaluated in osteoclasts differentiated from BMDMs. As shown in Figure 4F-J, FBXO9

216 knockdown significantly increased the $\mathrm{Fe}^{2+}$ level, MDA content, ROS level and decreased the

217 GPX4 level, GSH content in osteoclasts. These results suggested that FBXO9 inhibition facilitates

218 the ferroptosis of osteoclasts

\section{ZA treatment facilitated the ferroptosis of osteoclasts by suppressing FBXO9}

220 To explore whether FBXO9 mediated the function of ZA in regulating the ferroptosis of

221 osteoclasts, the osteoclasts differentiated from BMDMs were treated by ZA in the presence or

222 absence of FBXO9. As shown in Figure 5A and B, the qPCR and western blotting analysis results

223 showed that the expressions of FBXO9 were decreased by ZA treatment and restored by FBXO9

224 overexpression. CCK8 results showed that cell viability of osteoclasts was decreased by ZA

225 treatment, but these effects were blocked by FBXO9 overexpression (Figure 5C). Consistently, the

226 FDA staining also showed the inhibition of ZA on osteoclasts cell viability was restored by FBXO9

227 overexpression (Figure 5D and E). Besides, the $\mathrm{Fe}^{2+}$ level, MDA content, and ROS level were

228 obviously increased and the GPX4 level, GSH content was significantly decreased by ZA

229 treatment, while these effects were blocked by FBXO9 overexpression (Figure 5F-J). These results

230 suggested that ZA treatment facilitates the ferroptosis of osteoclasts by suppressing FBXO9.

231 FBXO9 inhibition facilitated the ferroptosis of osteoclasts by blocking the ubiquitin 
232

233

234

235

236

237

238

239

240

241

242

243

244

245

246

247

248

249

250

251

252

\section{mediated-proteasome degradation of $\mathrm{p53}$}

Previous studies showed that FBXO9, an E3 ubiquitin ligase, mediated protein stability through ubiquitin mediated-proteasome degradation[20]. Given that dysregulated ubiquitination has been widely reported to be involved in many diseases by regulating cell ferroptosis[26, 27], the target of FBXO9 was predicted by ubibrowser (http://ubibrowser.ncpsb.org.cn/ubibrowser/). Figure 6A showed the 20 potential target genes that interacted with FBXO9. Interestingly, among these genes, the p53 gene is an important regulator of ferroptosis. We next explored whether FBXO9 decreases p53 protein level by promoting its ubiquitination-mediated degradation. Knockdown of FBXO9 in osteoclasts did not change the p53 mRNA level (Figure 6B). Fascinatingly, the protein level of p53 was significantly increased after FBXO9 inhibition (Figure 6C), suggesting that FBXO9 decreased p53 expression possibly by the ubiquitin-proteasome-mediated degradation.

Next, a reciprocal Co-IP assay was performed to confirm whether FBXO9 directly interacts with p53. As shown in Figure 6D, a positive p53 signal was observed in the protein complex pulled down by FBXO9 antibody. Meanwhile, FBXO9 was also detected in the co-immunoprecipitation complex pulled-down by p53 antibody. Next, cycloheximide assay (CHX) was performed to detect the protein stability of p53 in osteoclasts transfected with si-FBXO9. As shown in Figure 6E, the protein stability of p53 was obviously increased in the FBXO9 knockdown osteoclasts. Then the p53 ubiquitination was assessed through IP with FBXO9 antibody and subsequent western blotting with ubiquitin antibody. Figure 6F showed that FBXO9 knockdown obviously decreased p53 ubiquitination in osteoclasts (Figure 6F). in conclusion, these results indicated that FBXO9 directly interacts with p53 and promotes its degradation. 
Discussion

BRONJ is one of the severe complications of BPs administration reported by Marx et al. in 2003[6]. It usually occurs in patients with bone metastatic cancer or osteoporosis, and undergoes bisphosphonate therapy. ZA is a kind of nitrogen-containing bisphosphonates and is widely used in the treatment of bone metastatic cancer and osteoporosis. Zhu et al. reported that ZA facilitates TLR-4-mediated M1 type macrophage polarization in the development of BRONJ[28]. Huang et al. demonstrated that ZA inhibited osteoclast differentiation and function by regulating the NF- $\mathrm{B}$ and JNK signaling pathways[29]. However, the mechanisms underlying ZA regulates osteoclast function in the occurrence of BRONJ remains unclear. In the current study, we clarified that ZA promotes osteoclasts ferroptosis by inhibiting FBXO9-mediated p53 ubiquitination and degradation, as evidence by (I) ZA treatment facilitated the ferroptosis of osteoclasts; (II) FBXO9 was downregulated in osteoclasts after ZA treatment; (III) FBXO9 inhibition facilitated the ferroptosis of osteoclasts; (IV) ZA treatment facilitated the ferroptosis of osteoclasts by suppressing FBXO9;(V) FBXO9 inhibition facilitated the ferroptosis of osteoclasts by blocking the ubiquitin mediated-proteasome degradation of p53.

Although a growing body of research have explored the role of BPs in the pathogenesis of BRONJ, the mechanism of BPs on the development of BRONJ is not completely understood. Growing studies have demonstrated that BPs have high affinity to hydroxyapatite crystals, thereby suppressing the osteoclasts resorptive ability by inducing the apoptosis of osteoclasts[30, 31$]$. 
274 was blocked, thus suppressing the healing ability of bone, suggesting that the differentiation of

275 osteoclasts plays an important role in the development of BRONJ[32]. More recently, ZA has

276 been reported to inhibit osteoclast differentiation by regulating the NF- $\kappa \mathrm{B}$ and JNK signaling

277 pathways[29]. Another study has shown that ZA inhibits osteoclast differentiation by interrupting

278 RANKL/RANK pathway[33]. Consistent with previous studies, we confirmed that ZA decreased

279 cell viability of osteoclasts induced by RANKL, specifically ZA-induced cell viability decrease

280 was blocked by ferroptosis inhibitor, suggesting an important role of ferroptosis in the

281 development of BRONJ.

282

Ferroptosis is a kind of iron- and ROS-dependent form of cell death, different with necrosis,

apoptosis, and other forms of cell death. Right now, almost all the mechanisms of ferroptosis are

associated with reactive oxygen species (ROS)[12]. Given the role of ZA in regulating ROS

production [22-24], here we investigated whether ZA suppresses the growth of osteoclast by

accelerating ferroptosis. We found that the ferroptosis-related marker such as the levels of $\mathrm{Fe}^{2+}$,

MDA content, ROS level was obviously increased in the osteoclasts treated with ZA, suggesting

the ZA induced the ferroptosis of osteoclasts. However, the underlying mechanism of ZA-induced

osteoclast ferroptosis reminds unknown.

To elucidate the mechanism of ZA-induced osteoclast ferroptosis, we compared the

expression profiles of osteoclasts in the presence or absence of alendronate or risedronate

treatment, and got 18 genes with significant differences in the osteoclasts treated by BPs. Among

these18 genes, FBXO9 was identified to be significantly reduced in ZA-treated osteoclasts.

Further experiment showed that FBXO9 knockdown promoted the ferroptosis of osteoclasts, and 
295

296

297

298

299

300

301

302

303

304

305

306

307

308

309

310

311

312

313

314

315

the ferroptosis of osteocalsts induced by ZA was blocked by FBXO9 overexpression, suggesting that ZA promotes the ferroptosis of osteoclasts by downregulating the expression of FBXO9.

The F-box only protein 9 (FBXO9), a member of the F-box protein family, is the substrate recognition subunit of skp1-cullin1-f-box E3 ligase complex and plays a key role in ubiquitination and subsequent target protein degradation[19]. Liu et al. demonstrated that FBXO9 interacted with Neurog2 and promoted its destabilization is a major contributor in directing multipotent NC progenitors toward glial lineage [20]. Vanesa Fernández-Sáiz et al. demonstrated that, under the growth factor deprivation condition, FBXO9-mediated ubiquitination of Tel2 and Tti1 inactivated mTORC1, but activated the PI3K/Akt pathway to increase survival of multiple myeloma[21]. However, the function of FBXO9 in the development of BRONJ and the regulatory mechanism remain unclear. Growing studies suggested that E3 ubiquitin ligase regulates ferroptosis by degrading substrates. Yang et al. reported that Nedd4 ubiquitylates VDAC2/3 to suppress erastininduced ferroptosis in melanoma[26]. Another study showed that TRIM26 facilitates the ferroptosis of HSCs to suppress liver fibrosis by mediating the ubiquitination of SLC7A11[35]. Therefore, we speculated whether FBXO9 also regulates ferroptosis by mediating the ubiquitination of target genes. Interestingly, we found that p53, a key upstream regulator of ferroptosis, is one of the FBXO9 targets. Our data showed that FBXO9-knowdown did not change the p53 mRNA level but significantly increased the p53 protein level, suggesting that FBXO9mediated p53 expression by the ubiquitin-proteasome system. Further experiment showed that FBXO9 directly interacts with p53 and the ubiquitination level of p53 was downregulated by FBXO9 knockdown. In addition, the protein stability of p53 was promoted by FBXO9 knockdown. 
316 These data suggesting that p53 is the direct target of FBXO9 and FBXO9-mediated p53

317 ubiquitination and degradation in osteoclast.

\section{Conclusions}

319 Taken together, the current data demonstrated that FBXO9 was downregulated in ZA-treated

320 osteoclast and promoted osteoclasts ferroptosis by inhibiting FBXO9-mediated p53 ubiquitination

321 and degradation. Our study provided a possible theoretical target for the clinical treatment of

\section{BRONJ.}

323 There are still some deficiencies in the current research, such as the current conclusions still need

324 to be further confirmed by clinical and animal experiments.

\section{Conflicts of Interest}

326 The authors declare no competing or financial interests.

\section{References}

1. Zhang, S., G. Gangal, and H. Uludag, 'Magic bullets' for bone diseases: progress in rational design of boneseeking medicinal agents. Chem Soc Rev, 2007. 36(3): p. 507-31.

2. Black, D.M., et al., Atypical Femur Fractures: Review of Epidemiology, Relationship to Bisphosphonates, Prevention, and Clinical Management. Endocr Rev, 2019. 40(2): p. 333-368.

3. La-Beck, N.M., et al., Repurposing amino-bisphosphonates by liposome formulation for a new role in cancer treatment. Semin Cancer Biol, 2021. 68: p. 175-185.

4. $\quad$ Favus, M.J., Bisphosphonates for osteoporosis. N Engl J Med, 2010. 363(21): p. 2027-35.

5. Mhaskar, R., et al., Bisphosphonates in multiple myeloma: an updated network meta-analysis. Cochrane Database Syst Rev, 2017. 12: p. CD003188.

6. Marx, R.E., Pamidronate (Aredia) and zoledronate (Zometa) induced avascular necrosis of the jaws: a growing epidemic. J Oral Maxillofac Surg, 2003. 61(9): p. 1115-7.

7. Maciel, A.P., et al., Clinical profile of individuals with bisphosphonate-related osteonecrosis of the jaw: an integrative review. Sao Paulo Med J, 2020. 138(4): p. 326-335.

8. Hadjidakis, D.J. and Androulakis, II, Bone remodeling. Ann N Y Acad Sci, 2006. 1092: p. $385-96$.

9. Ikebuchi, Y., et al., Coupling of bone resorption and formation by RANKL reverse signalling. Nature, 2018. 
561(7722): p. 195-200.

10. Madel, M.B., et al., Immune Function and Diversity of Osteoclasts in Normal and Pathological Conditions. Front Immunol, 2019. 10: p. 1408.

11. Wang, L., et al., Various pathways of zoledronic acid against osteoclasts and bone cancer metastasis: a brief review. BMC Cancer, 2020. 20(1): p. 1059.

12. Li, J., et al., Ferroptosis: past, present and future. Cell Death Dis, 2020. 11(2): p. 88.

13. Hirschhorn, T. and B.R. Stockwell, The development of the concept of ferroptosis. Free Radic Biol Med, 2019. 133: p. 130-143.

14. Mou, Y., et al., Ferroptosis, a new form of cell death: opportunities and challenges in cancer. J Hematol Oncol, 2019. 12(1): p. 34.

15. Reichert, C.O., et al., Ferroptosis Mechanisms Involved in Neurodegenerative Diseases. Int J Mol Sci, 2020. 21(22).

16. Sun, Y., et al., The emerging role of ferroptosis in inflammation. Biomed Pharmacother, 2020. 127: $\mathrm{p}$. 110108.

17. Bagan, J., et al., Oxidative stress in bisphosphonate-related osteonecrosis of the jaws. J Oral Pathol Med, 2014. 43(5): p. 371-7.

18. Ma, H., et al., Melatonin Suppresses Ferroptosis Induced by High Glucose via Activation of the Nrf2/HO-1 Signaling Pathway in Type 2 Diabetic Osteoporosis. Oxid Med Cell Longev, 2020. 2020: p. 9067610.

19. Lee, K.W., et al., F-box only protein 9 is an E3 ubiquitin ligase of PPARgamma. Exp Mol Med, 2016. 48: p. e234.

20. Liu, J.A., et al., Fbxo9 functions downstream of Sox10 to determine neuron-glial fate choice in the dorsal root ganglia through Neurog2 destabilization. Proc Natl Acad Sci U S A, 2020. 117(8): p. 4199-4210.

21. Fernandez-Saiz, V., et al., SCFFbxo9 and CK2 direct the cellular response to growth factor withdrawal via Tel2/Tti1 degradation and promote survival in multiple myeloma. Nat Cell Biol, 2013. 15(1): p. 72-81.

22. Sehitoglu, I., et al., Zoledronic acid aggravates kidney damage during ischemia reperfusion injury in rat. J Environ Pathol Toxicol Oncol, 2015. 34(1): p. 53-61.

23. Wang, L., et al., The apoptotic effect of Zoledronic acid on the nasopharyngeal carcinoma cells via ROS mediated chloride channel activation. Clin Exp Pharmacol Physiol, 2018. 45(10): p. 1019-1027.

24. Liu, L., et al., Zoledronic Acid Enhanced the Antitumor Effect of Cisplatin on Orthotopic Osteosarcoma by ROS-PI3K/AKT Signaling and Attenuated Osteolysis. Oxid Med Cell Longev, 2021. 2021: p. 6661534.

25. Takahashi, N., et al., Generating murine osteoclasts from bone marrow. Methods Mol Med, 2003. 80: p. 129-44.

26. Yang, Y., et al., Nedd4 ubiquitylates VDAC2/3 to suppress erastin-induced ferroptosis in melanoma. Nat Commun, 2020. 11(1): p. 433.

27. Zhang, Z., et al., RNA-binding protein ZFP36/TTP protects against ferroptosis by regulating autophagy signaling pathway in hepatic stellate cells. Autophagy, 2020. 16(8): p. 1482-1505.

28. Zhu, W., et al., Zoledronic acid promotes TLR-4-mediated M1 macrophage polarization in bisphosphonaterelated osteonecrosis of the jaw. FASEB J, 2019. 33(4): p. 5208-5219.

29. Huang, X.L., et al., Zoledronic acid inhibits osteoclast differentiation and function through the regulation of NF-kappaB and JNK signalling pathways. Int J Mol Med, 2019. 44(2): p. 582-592.

30. Favia, G., et al., Medication-related osteonecrosis of the jaw: Surgical or non-surgical treatment? Oral Dis, 
2018. 24(1-2): p. 238-242.

31. Russell, R.G., Bisphosphonates: the first 40 years. Bone, 2011. 49(1): p. 2-19.

32. AlDhalaan, N.A., A. BaQais, and A. Al-Omar, Medication-related Osteonecrosis of the Jaw: A Review. Cureus, 2020. 12(2): p. e6944.

33. Li, M., et al., Regulation of osteogenesis and osteoclastogenesis by zoledronic acid loaded on biodegradable magnesium-strontium alloy. Sci Rep, 2019. 9(1): p. 933.

34. Tamaoka, J., et al., Osteonecrosis of the jaws caused by bisphosphonate treatment and oxidative stress in mice. Exp Ther Med, 2019. 17(2): p. 1440-1448.

35. Zhu, Y., et al., TRIM26 Induces Ferroptosis to Inhibit Hepatic Stellate Cell Activation and Mitigate Liver Fibrosis Through Mediating SLC7A11 Ubiquitination. Front Cell Dev Biol, 2021. 9: p. 644901.

\section{Figure legends}

\section{Figure 1.ZA treatment facilitated the ferroptosis of osteoclasts}

The osteoclasts cell model induced by RANKL (50 ng/ml) treatment. (A and B) Multinucleated cells were visualized by tartrate-resistant acid phosphatase (TRAP) staining.(C and D) Cell viability of Raw264.7 and BMDM derived osteoclasts was assessed using CCK8 assay after treatment with different concentrations of ZA $(5,10$, and $50 \mu \mathrm{M})(\mathrm{n}=3)$. (E and F) Cell viability of Raw264.7 and BMDM derived osteoclasts was assessed using FDA staining after treatment with different concentrations of ZA $(5,10$, and $50 \mu \mathrm{M})(\mathrm{n}=3)$. ( $\mathrm{G}$ and $\mathrm{H})$ Cell viability of Raw264.7 and BMDM derived osteoclasts was assessed using CCK8 assay after treatment with ZA for $48 \mathrm{~h}(50 \mu \mathrm{M})$ in the presence or absence of $10 \mu \mathrm{M}$ of ZVAD-FMK, $2 \mu \mathrm{M}$ of Fer- 1 , or $10 \mu \mathrm{M}$ of necrostatin-1(n=3). ${ }^{*} p<0.05, * * p<0.01$.

Figure 2. ZA treatment facilitated the ferroptosis of osteoclasts

(A-E) the level of $\mathrm{Fe}^{2+}$, MDA content, ROS level, the level of Gpx4, and GSH content in Raw264.7 derived osteoclasts was assessed by Elisa assay after treatment with different concentrations of ZA $(5,10$, and $50 \mu \mathrm{M})(\mathrm{n}=3) * p<0.05, * * p<0.01, * * * p<0.001$. (F-J) the level of $\mathrm{Fe}^{2+}$, MDA content, 
411 ROS level, the level of Gpx4, and GSH content in BMDM derived osteoclasts was assessed by

412 Elisa assay after treatment with different concentrations of ZA $(5,10$, and $50 \mu \mathrm{M})(\mathrm{n}=3) * p<0.05$, $413 * * p<0.01, * * * p<0.001$.

414 Figure 3. FBXO9 was downregulated in osteoclasts after ZA treatment

415 (A) Venn analysis of DEGs of alendronate and risedronate-treated osteoclast. (B and C) The 416 mRNA level of 18 genes in Raw264.7 and BMDM derived osteoclasts was assessed using qPCR 417 after treatment with ZA $(50 \mu \mathrm{M})(\mathrm{n}=3) * p<0.05, * * p<0.01$. (D) The protein level of FBXO9 in 418 Raw264.7 and BMDM derived osteoclasts was assessed using western blot after treatment with 419 ZA $(50 \mu \mathrm{M})$

Figure 4. FBXO9 inhibition facilitated the ferroptosis of osteoclasts

(A) The mRNA level of FBXO9 in BMDM derived osteoclasts was assessed using qPCR after

treatment with or without si-FBXO9 $(\mathrm{n}=3) .{ }^{* * *} p<0.001$. (B) The protein level of FBXO9 in

BMDM derived osteoclasts was assessed using western blot after treatment with or without si-

FBXO9 ( $\mathrm{n}=3) . * * p<0.01$. (C)Cell viability of BMDM derived osteoclasts was assessed using

CCK8 assay after treatment with or without si-FBXO9 $(\mathrm{n}=3) . * * p<0.01$. (D and E) Cell viability

of BMDM derived osteoclasts was assessed using FDA staining after treatment with or without si-

FBXO9 (n=3). ${ }^{*} p<0.05$. (F-J) the level of $\mathrm{Fe}^{2+}$, MDA content, ROS level, the level of Gpx4, and

GSH content in BMDM derived osteoclasts was assessed by Elisa assay after treatment with or without si-FBXO9 $(\mathrm{n}=3) * p<0.05, * * p<0.01$.

Figure 5. ZA treatment facilitated the ferroptosis of osteoclasts by suppressing FBXO9

(A) The mRNA level of FBXO9 in BMDM derived osteoclasts was assessed using qPCR after 
432 433 434 435 436 437 438 439 440

treatment with ZA $(50 \mu \mathrm{M})$ in the presence or absence of FBXO9 $(n=3)$. $* * p<0.01$. (B) The protein level of FBXO9 in BMDM derived osteoclasts was assessed using western blot after treatment with ZA $(50 \mu \mathrm{M})$ in the presence or absence of FBXO9 $(\mathrm{n}=3) . * p<0.05$. (C) Cell viability of BMDM derived osteoclasts was assessed using CCK8 assay after treatment with ZA $(50 \mu \mathrm{M})$ in the presence or absence of FBXO9 $(\mathrm{n}=3) .{ }^{*} p<0.05, * * p<0.01$. (D and E) Cell viability of BMDM derived osteoclasts was assessed using FDA staining after treatment with ZA $(50 \mu \mathrm{M})$ in the presence or absence of FBXO9 (n=3). * ${ }^{*}<0.05$. (F-J) the level of $\mathrm{Fe}^{2+}$, MDA content, ROS level, the level of Gpx4, and GSH content in BMDM derived osteoclasts was assessed by Elisa assay after treatment with ZA $(50 \mu \mathrm{M})$ in the presence or absence of FBXO9 $(\mathrm{n}=3) * p<0.05, * * p<0.01$.

\section{Figure 6. FBXO9 inhibition facilitated the ferroptosis of osteoclasts by blocking the ubiquitin}

\section{mediated-proteasome degradation of p53}

(A) The target of FBXO9 was predicted by ubibrowser. (B) the p53 mRNA expression in the FBXO9 knockdown and control cell was assessed by qPCR $(n=3)$. (C) the protein level of p53 in the FBXO9 knockdown and control cell was assessed by western blot (n=3). (D) FBXO9 directly interacts with $\mathrm{p} 53$. The proteins from BMDM derived osteoclasts were IP with IgG or antibodies against FBXO9 and p53, following by western blot analysis $(n=3)$. (E) The stability of p53 protein was regulated by $\mathrm{FBXO9.} \mathrm{BMDM}$ derived osteoclasts treated with or without si-FBXO9 in the presence of cycloheximide $(\mathrm{CHX}, 25 \mathrm{ug} / \mathrm{ml})$ for various times as indicated and cell lysates were then assessed by western blot $(\mathrm{n}=3) .{ }^{*} p<0.01$. (F) The cell lysates isolated from scramble and siFBXO9 infected BMDM derived osteoclasts were immunoprecipitated with anti-p53 antibody, then analyzed by western blot using ubiquitin antibody $(n=3)$. 
Table $\mathbf{1}$ (on next page)

sequence

sequence 
1 CFAP53:

2 forward primer:5'-GACAAAATGAGAGAGAGAACCAAGT-3'

3 reverse primer: 5'-TCCCTGAACTGCTGGTCTAAC-3'

4 COL14A1

5 forward primer:5'-ACTGGTTTTCACGGGTGTTC-3',

6 reverse primer: 5'-TAAGTCGAGGAGAGGCAAGC-3'

7 ARSJ

8 forward primer:5'- CTGAGATAAAGACGCCCACC-3',

9 reverse primer: 5'- ATAGAATGCTGAAGTCCCGTG-3'

10 ABCA9

11 forward primer:5'- CAGAGGGAGTGAAGAGAAAGC -3',

12 reverse primer: 5'- GCTCTGTGTTTGTGAAAGTGG -3'

13 CXorf57:

14 forward primer:5'- GCAGTATAGGGAACAAAAGCG-3',

15 reverse primer: 5'- TGCTTGAGATGTTGAGGGAC-3'

16 GPR22:

17 forward primer:5'- CCACTGTCATACCCACTAAGC-3',

18 reverse primer: 5'- ATGCAGTAAAGTACCAGGACG-3'

19 STXBP5L:

20 forward primer:5'- GATCAAGTGACCTGTACCAGC-3',

21 reverse primer: 5'- ATTTACATGGTCTGAGGTGGG-3'

22 MSANTD4

23 forward primer:5'- CAGAGGTCAAAGTGGAAGAGG-3',

24 reverse primer: 5'- ATCAATGTGAGGGAAGTCAGG-3'

25 RRP15

26 forward primer:5'- GAAATGCTGTGCAGAGTGAAG-3',

27 reverse primer: 5'- TCCTGCTTCCTTAACCTTTTCG-3'

28 UGT1A2

29 forward primer:5'- TCTGCGTTCTCTTTCCTGTG -3',

30 reverse primer: $5^{\prime}$ - AGCATGTTCTGGACCCTTTG $-3^{\prime}$

31 IRF4

32 forward primer:5'- AACAAGCTAGAAAGAGACCAGAC-3',

33 reverse primer: 5'- TCACCAAAGCACAGAGTCAC-3'

34 TFAP2D

35 forward primer:5'- AAAGATGATCCTAGCCACCAAG-3',

36 reverse primer: $5^{\prime}$ - TGTGTTAAGTGCCTCTGGATG-3'

37 TRHDE

38 forward primer:5'- AGGAAGGCTTTGCTCACTAC -3',

39 reverse primer: 5'- CTGTGATACTGGATGGGAACTG -3'

40 ASMT

41 forward primer:5'- GAAGTGGGACAGGAAGTGAG -3', 
42 reverse primer: 5'- CGGGAACAGGAAGTGGC -3'

43 CAPS

44 forward primer:5'- AGCTCGAAGACACAATCCG -3',

45 reverse primer: 5'- TCCATGTCCACTGCAAAGAG -3'

46 COMMD10

47 forward primer:5'- AGTGGGATGGCAGCTTAAC-3',

48 reverse primer: 5'- TCGAACAGCTCCTTGTGATTG-3'

49 VSTM4

50 forward primer:5'- CCTGGCAGTCTGTGTTTCA-3',

51 reverse primer: 5'- CTCTTACCCTTCTGTGGCTG-3'

52 FBXO9

53 forward primer:5'- ATGAGAGTCCGGCTGAGAGA-3',

54 reverse primer: 5'- AGAGCTTCTTCCTGCTCTGC-3'

$5518 \mathrm{~s}$

56 forward primer:5'- CTCAACACGGGAAACCTCAC-3',

57 reverse primer: 5'- CGCTCCACCAACTAAGAACG-3' 
Figure 1

ZA treatment facilitated the ferroptosis of osteoclasts

ZA treatment facilitated the ferroptosis of osteoclasts 
A
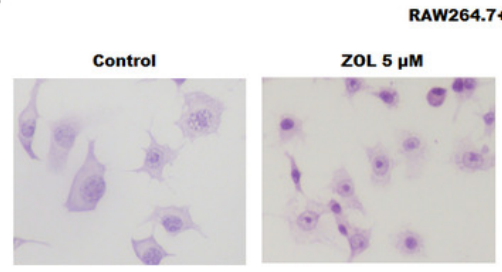

ZOL $10 \mu \mathrm{M}$

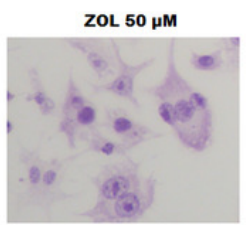

B
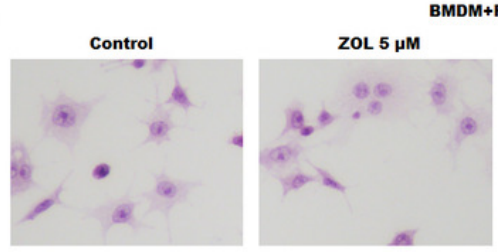

ZOL $10 \mu \mathrm{M}$

ZOL $50 \mu \mathrm{M}$

C
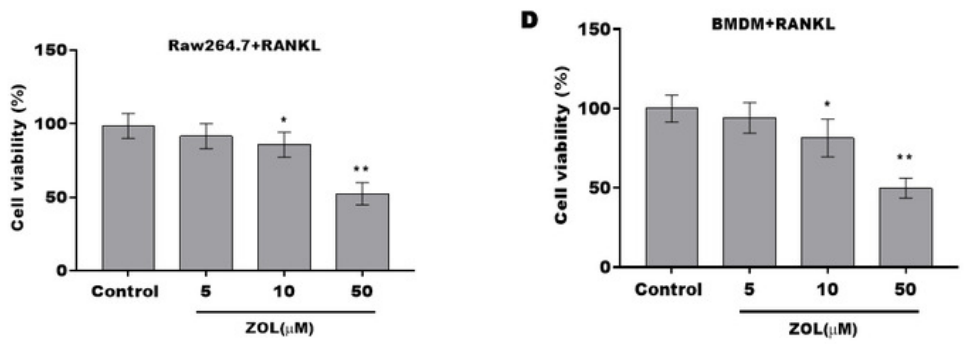

E
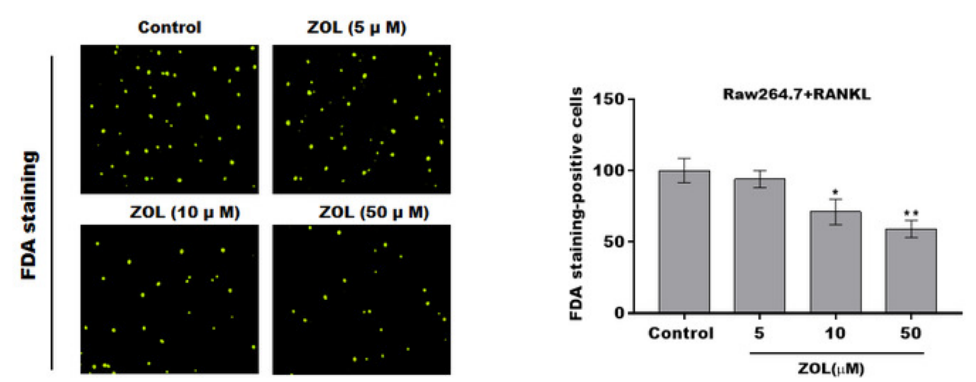

$\mathbf{F}$
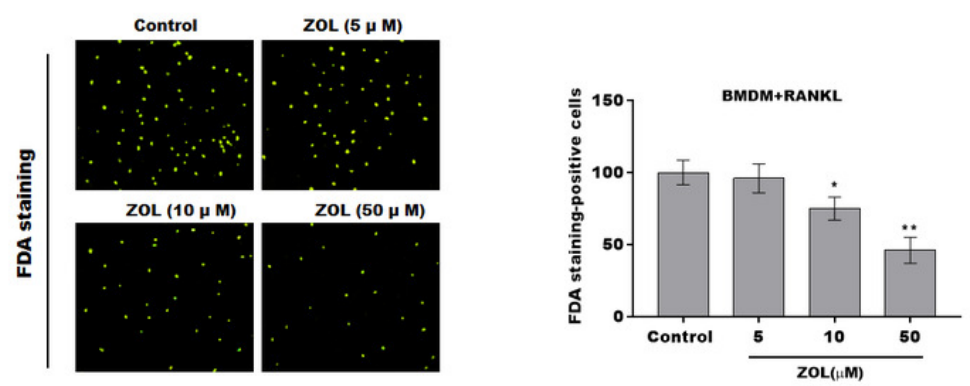

G

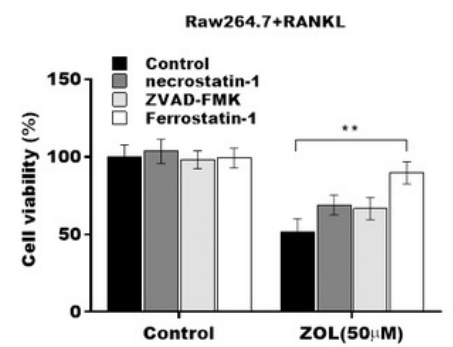

H

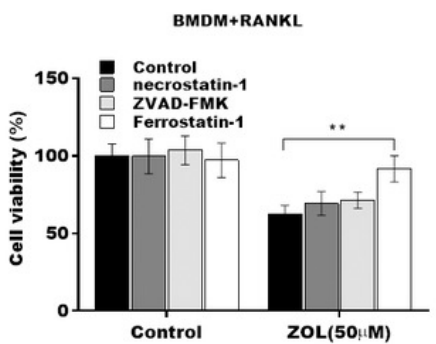


Figure 2

ZA treatment facilitated the ferroptosis of osteoclasts

\section{ZA treatment facilitated the ferroptosis of osteoclasts}
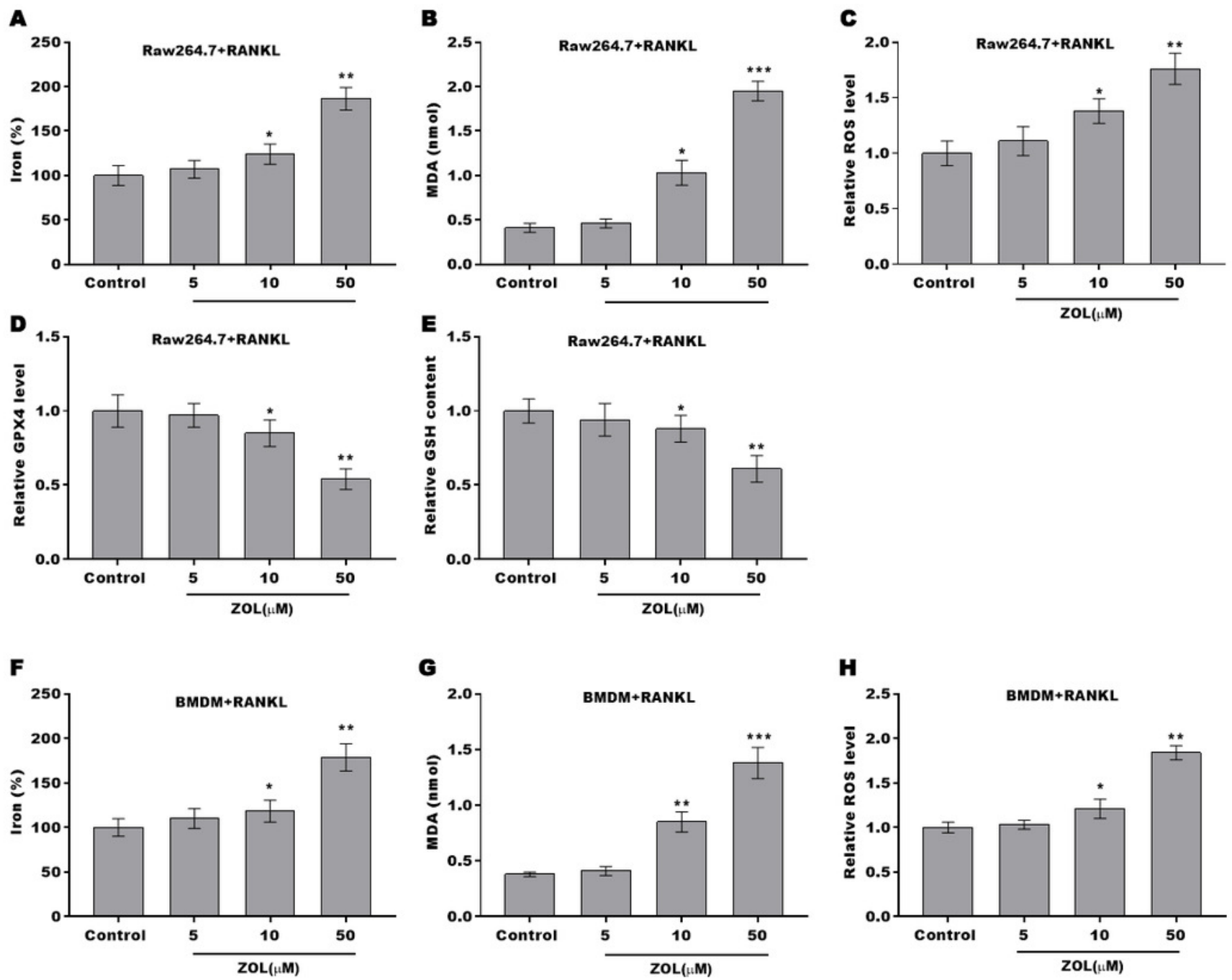

G

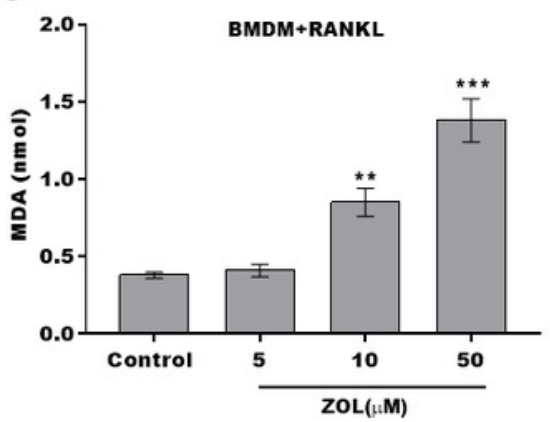

H
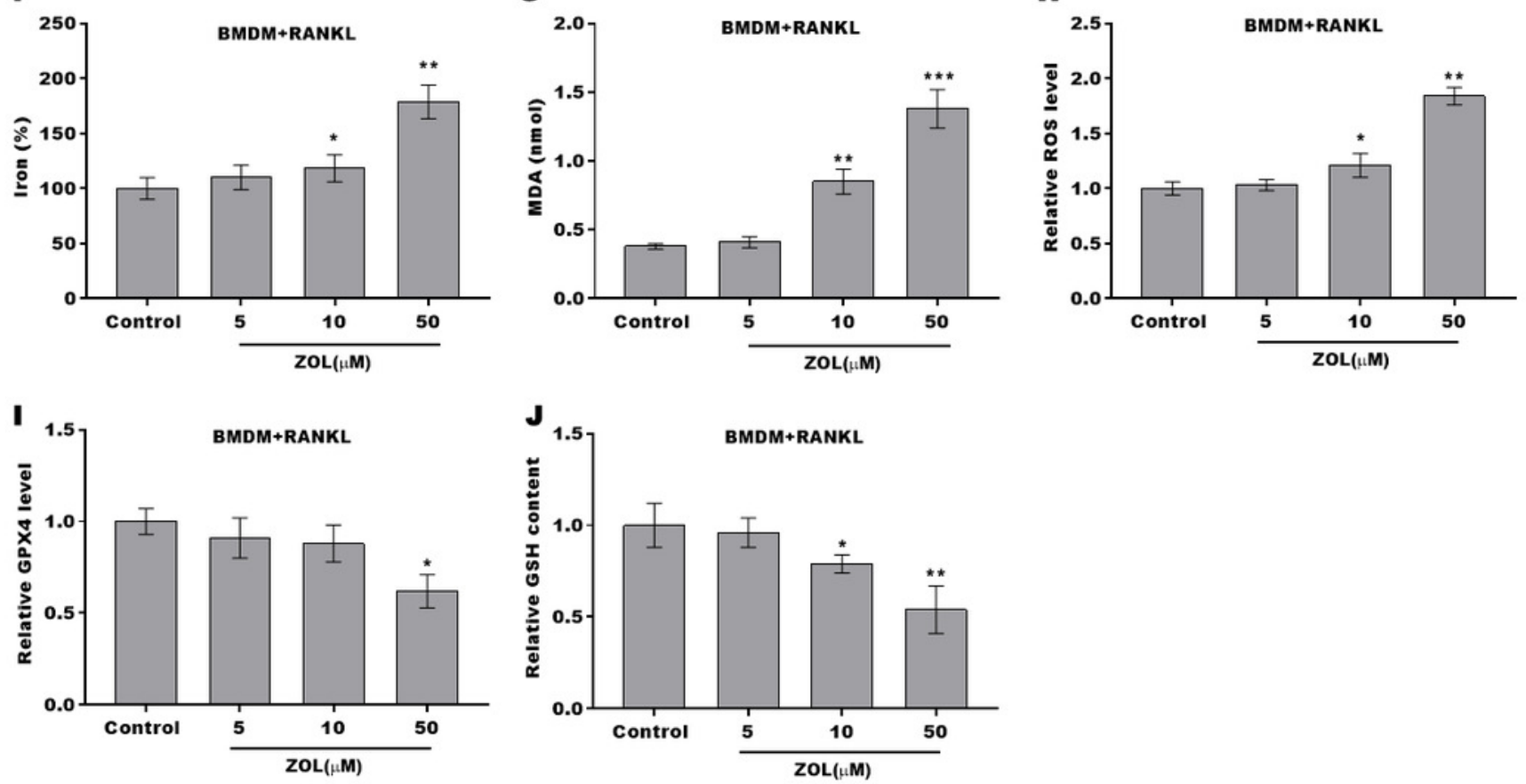
Figure 3

FBX09 was downregulated in osteoclasts after ZA treatment

FBX09 was downregulated in osteoclasts after ZA treatment

A

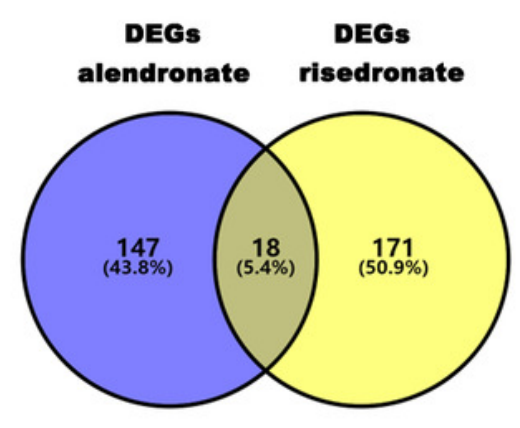

c

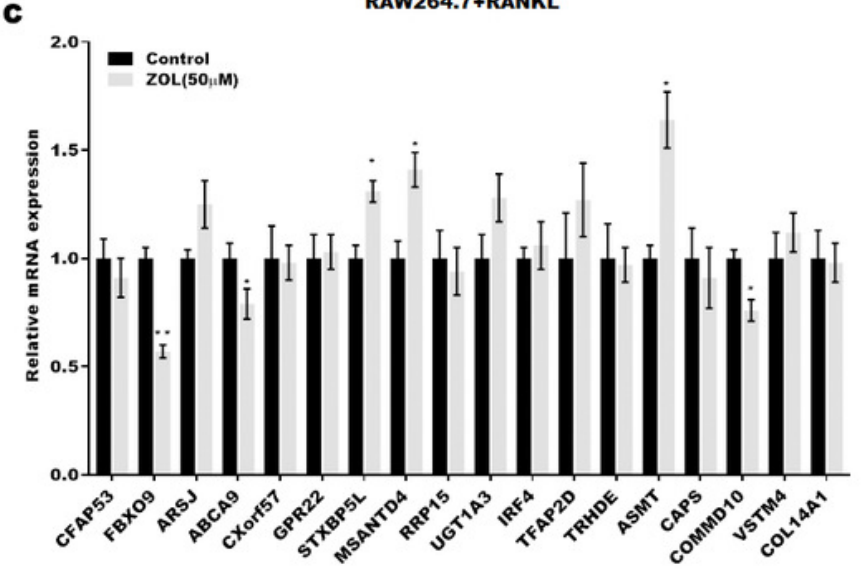

B

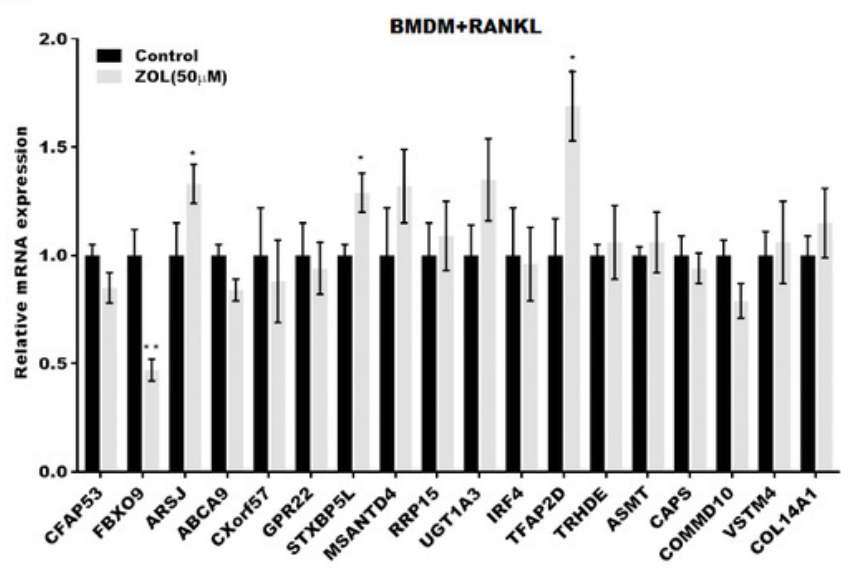

D

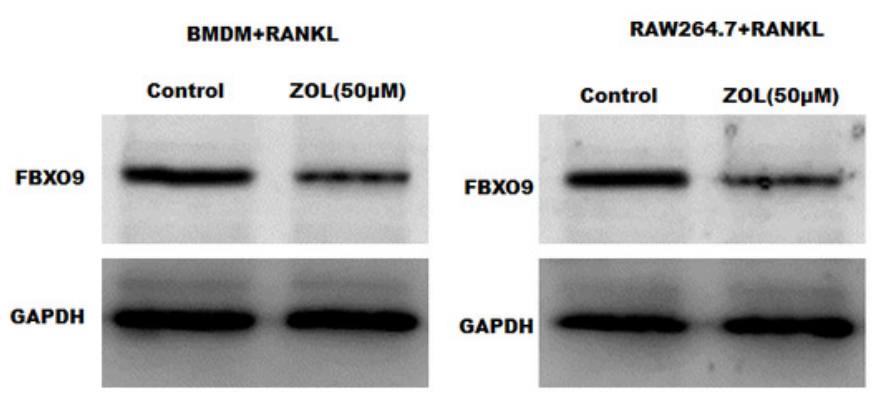


Figure 4

FBXO9 inhibition facilitated the ferroptosis of osteoclasts

FBX09 inhibition facilitated the ferroptosis of osteoclasts

A

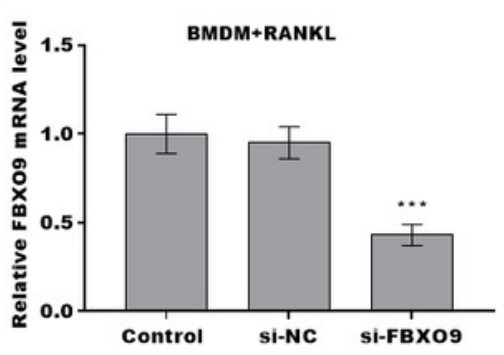

c

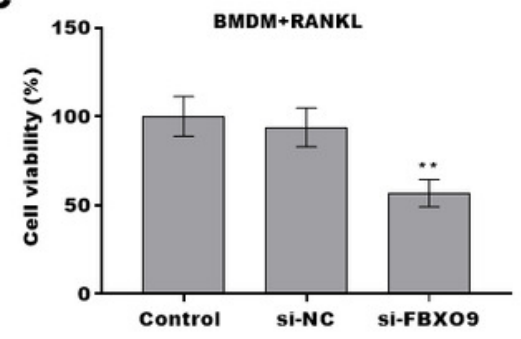

E

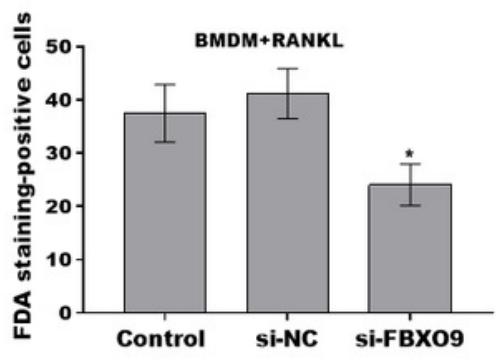

$\mathbf{H}$

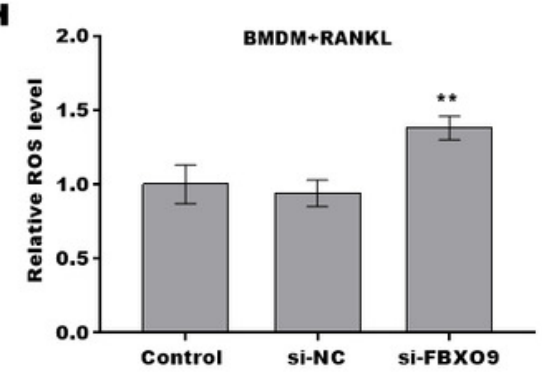

B

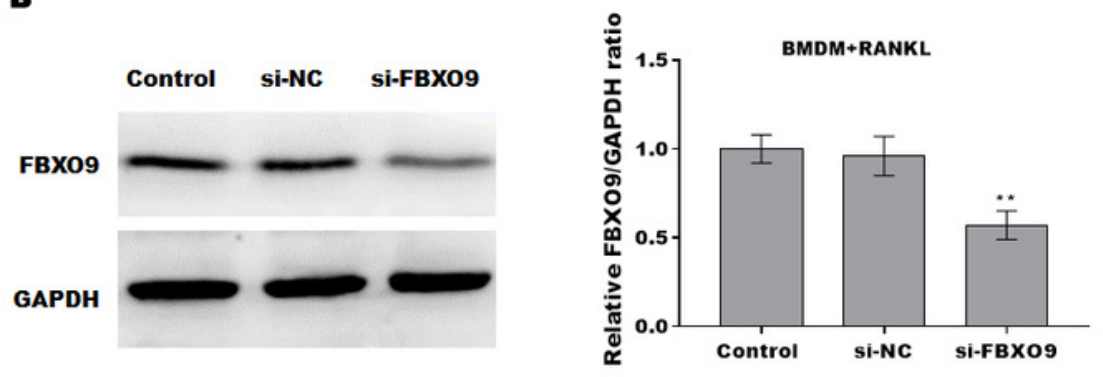

D

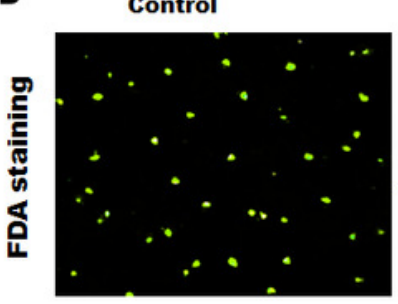

si-NC

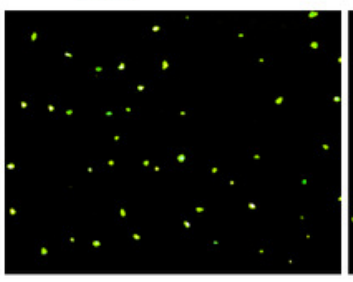

si-FBX09

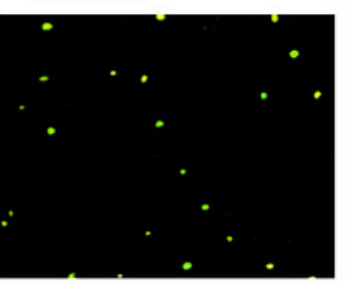

F

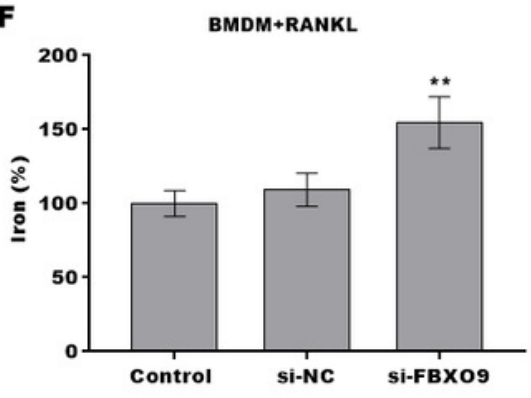

C
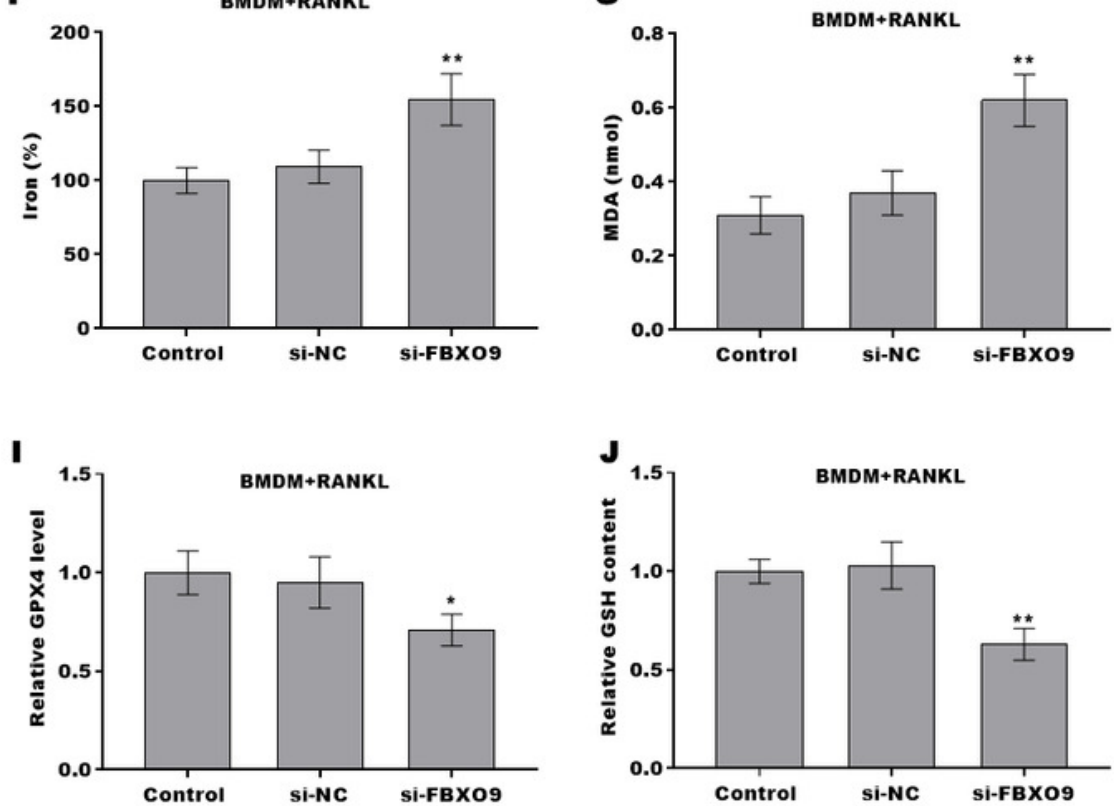


\section{Figure 5}

ZA treatment facilitated the ferroptosis of osteoclasts by suppressing FBXO9

\section{ZA treatment facilitated the ferroptosis of osteoclasts by suppressing FBX09}

A
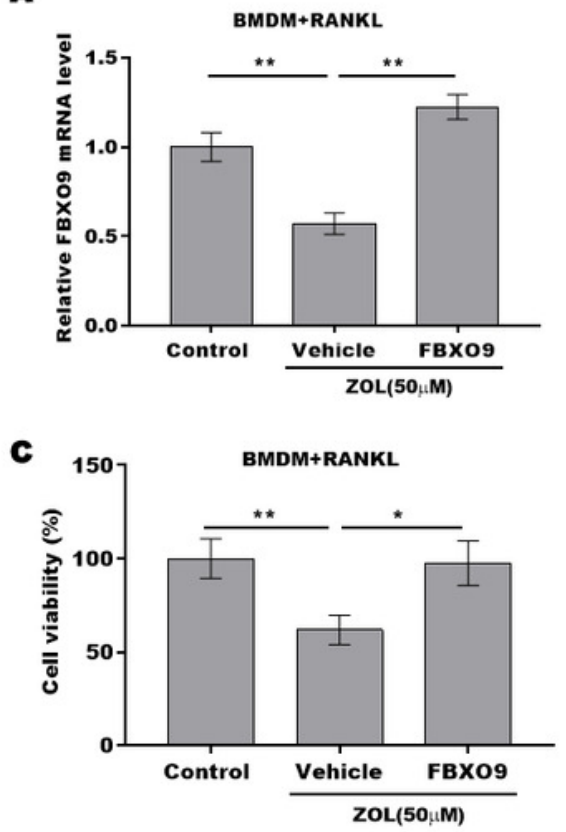

E

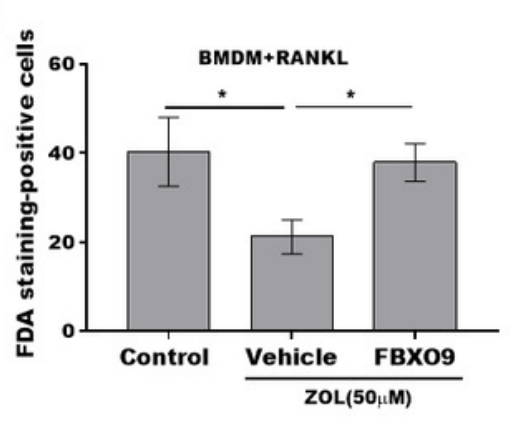

H

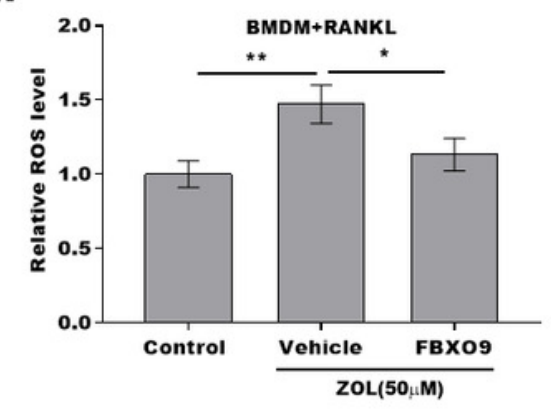

$\mathbf{B}$
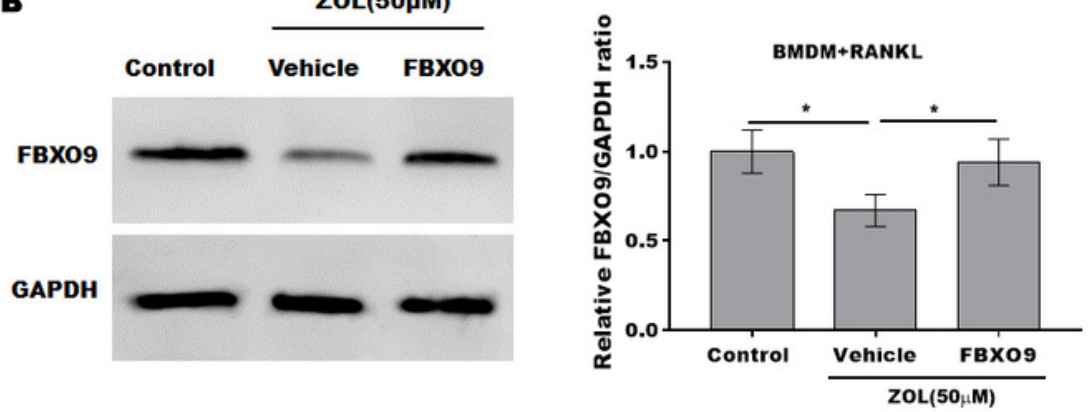

D
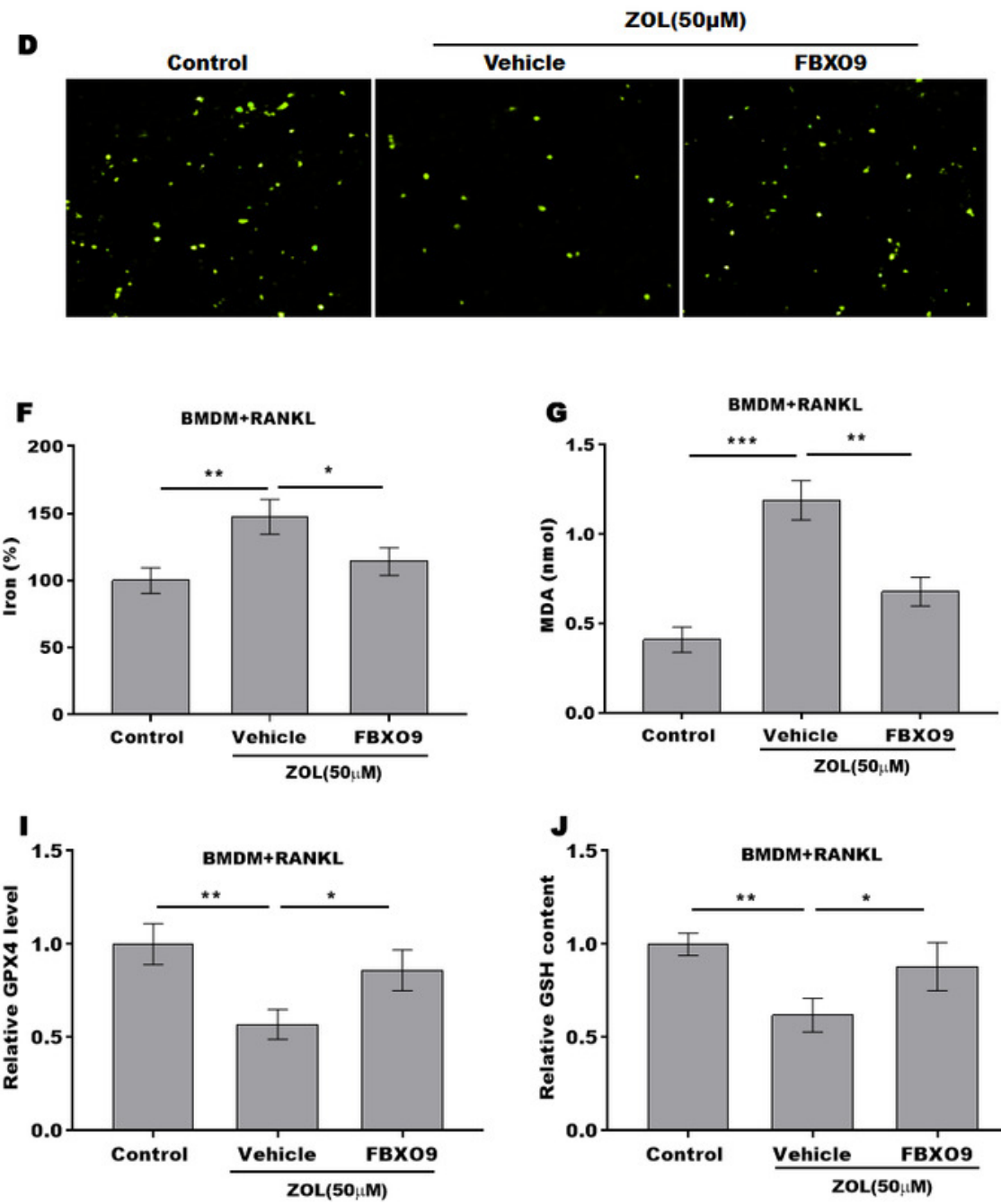
Figure 6

FBXO9 inhibition facilitated the ferroptosis of osteoclasts by blocking the ubiquitin mediated-proteasome degradation of p53

FBX09 inhibition facilitated the ferroptosis of osteoclasts by blocking the ubiquitin mediated-proteasome degradation of p53

A

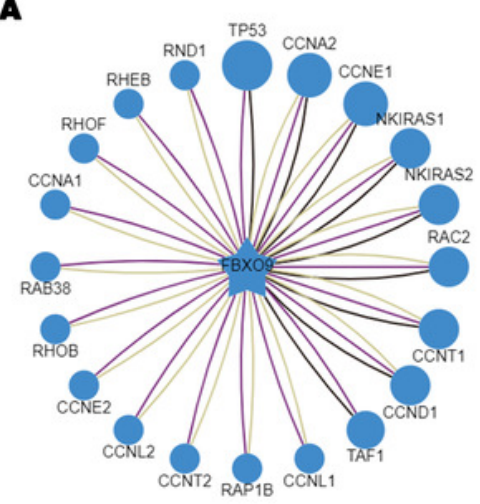

D

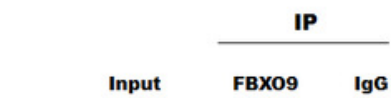

FBX09

P53
B
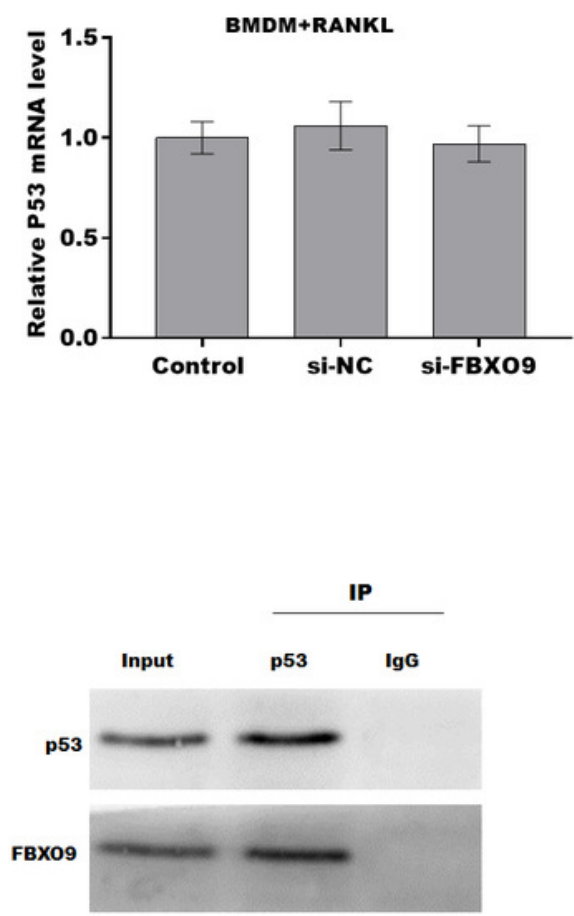

C
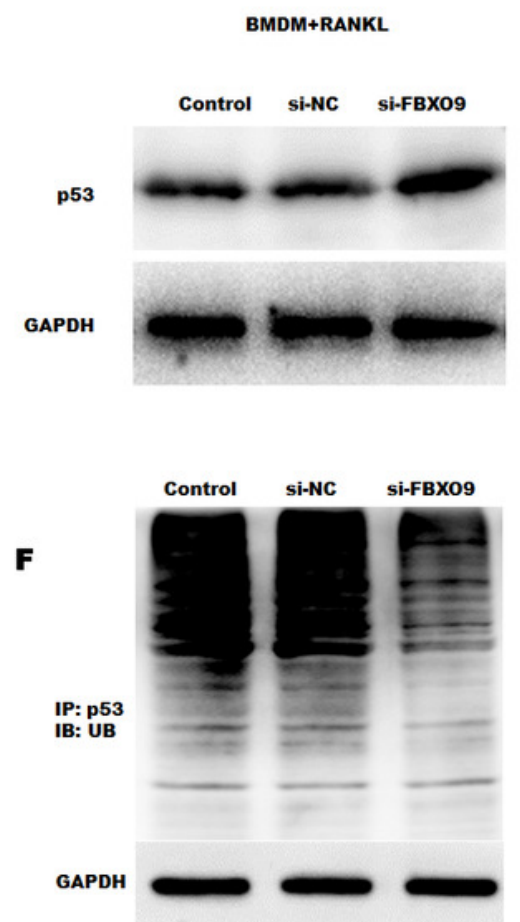

$\mathbf{E}$
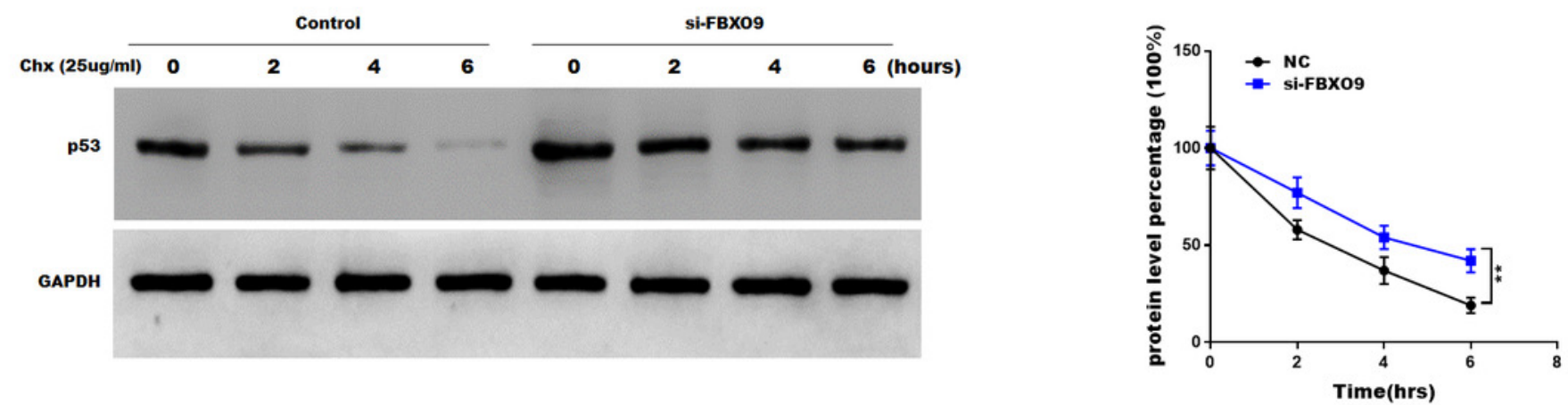